\title{
La competencia matemática en Educación Primaria mediante el aprendizaje basado en proyectos
}

\section{Mathematical competence in Primary Education by problem based learning}

\author{
Ane Izagirre ${ }^{1}$ \\ Lidia Caño \\ Andoni Arguiñano 3
}

Resumen: El presente artículo pretende demostrar que mediante el aprendizaje
basado en proyectos se puede desarrollar adecuadamente la competencia mate-
mática, así como responder a los contenidos curriculares. Para tal fin se optó por
realizar un estudio de carácter cualitativo siguiendo el método de estudio de
casos y las técnicas de recogida de información empleadas son la observación
participante y el análisis de las producciones del alumnado. El proyecto con-
siderado se ha llevado a cabo con seis niñas y doce niños de 6 o de primaria
de la escuela Antzuola Herri Eskola y trata el tema de la Cocina. El alumnado
acordó confeccionar algunos delantales para cocinar y pensaron calcular la
cantidad de tela necesaria para su elaboración. Sin embargo, el propósito se
vio modificado debido a la falta de tiempo y se muestra únicamente el cálcu-
lo del área de una manga. Tomando como referencia este estudio se

Fecha de recepción: 06 de abril de 2019. Fecha de aceptación: 10 de septiembre de 2020.

1 Universidad del País Vasco (UPV-EHU), Facultad de Educación, Filosofía y Antropología, ane.izagirre@ ehu.eus, orcid.org/0000-0001-8900-1576

2 Universidad del País Vasco (UPV-EHU), Facultad de Educación, Filosofía y Antropología, lidia.cano@ ehu.eus, orcid.org/0000-0002-3456-861X

3 Universidad del País Vasco (UPV-EHU), Facultad de Educación y Deporte, andoni.arguinano@ehu.eus, orcid.org/0000-0002-9585-8303 
demuestra que mediante el aprendizaje basado en proyectos se adquiere la competencia matemática y se cumple el currículo de educación.

Palabras clave: Competencia matemática, aprendizaje basado en proyectos, educación primaria, área, superficies planas.

\begin{abstract}
The aim of this article is to demonstrate that through project-based learning, it is possible to develop mathematical competence and respond to curricular content. For this purpose, a qualitative study has been carried out following the case study method. The data collection techniques used are participant observation and students' productions analysis. The project considered has been carried out with six girls and twelve boys from 6th grade of primary of Antzuola Herri Eskola school. The students agreed to assemble some aprons to cook with and they thought to work out the amount of fabric needed to make the aprons. However, due to lack of time, they only calculated the area of a sleeve. Taking this project as a reference, we demonstrate that through project-based learning, students develop the mathematical competence and the education curriculum is fulfilled.
\end{abstract}

Keywords: mathematical competence, project based learning, primary education, area, plane surfaces.

\title{
INTRODUCCIÓN
}

El presente artículo forma parte de un estudio más amplio (Antzuola: pasado, presente y futuro) centrado en el proyecto educativo que la escuela pública de Antzuola (en adelante Antzuola H.E.) ha desarrollado durante los últimos 40 años. Su práctica didáctica se lleva a cabo a partir de proyectos que proponen y exponen los/las propios/as estudiantes (Antzuola Herri Eskola, 2006; Sasieta y Etxaniz, 2015), cumpliendo las características esenciales del Project Based Learning (en adelante PBL) (Walker, 2015).

Las matemáticas están muy presentes en los proyectos de trabajo de Antzuola H.E. Por esta razón, queremos mostrar que es posible aprender matemáticas en un contexto donde el alumnado sea el protagonista del aprendizaje adoptando una actitud activa al tomar sus propias decisiones y que es capaz de definir su 
propio camino superando las distintas situaciones-problema que se le plantean; y todo ello respondiendo al currículo. El concepto de situación-problema desarrollado por Perrenoud se entiende que es "un problema en el que un alumno o alumna, individualmente o en grupo, deberá articular un conjunto contextualizado de informaciones a fin de resolver una tarea determinada en la que la solución no es evidente a priori" (Dpto. de Educación, Política Lingüística y Cultura, s.f-a, p. 3).

La situación-problema que se presenta en este estudio se desarrolla en el proyecto nombrado "Cocina". El alumnado, con el objetivo de preparar algunas recetas, acordó que sería buena idea confeccionar delantales y pensaron calcular la cantidad de tela necesaria para su elaboración. Sin embargo, el propósito se vio modificado debido al tiempo que requería el cálculo del área total de los delantales y se mostrará únicamente el cálculo del área de una manga. Basándose en este proyecto, se demuestra que mediante el aprendizaje basado en proyectos se puede desarrollar adecuadamente la competencia matemática y dar respuesta a los contenidos curriculares. Para este fin, se toma en consideración todo el proceso de aprendizaje y, en particular, se pone el foco en el cálculo del área de la manga, una tarea rica para el aprendizaje del concepto de área e introducción al cálculo del área de superficies planas. Para mostrar cómo inician los estudiantes el cálculo del área de una figura plana se presentan y analizan las diversas estrategias que emplean.

Es muy común el sentimiento de miedo y falta de confianza, entre el profesorado, a la hora de implementar la metodología PBL. Este artículo ofrece al profesorado de Educación Primaria un ejemplo real para el aprendizaje de las matemáticas que se podría implementar adaptándolo a la situación de cada aula.

\section{MARCO TEÓRICO}

El plan educativo de la Unión Europea parte desde una concepción del aprendizaje basado en las competencias tal y como se estipula en el artículo 2006/962/ CE del Parlamento Europeo (Recomendación del Parlamento Europeo y del Consejo, de 18 de diciembre de 2006, sobre las competencias clave para el aprendizaje permanente 2006/962/CE, 2006); de poco sirve el aprendizaje si no somos capaces de aplicarlo y experimentarlo en la situación o momento requerido. El plan educativo actual de la Comunidad Autónoma Vasca, Heziberri 2020 (Dpto. de Educación, Política Lingüística y Cultura, s.f.-b), como el nacional Ley Orgánica 
8/2013, de 9 de diciembre, para la Mejora de la Calidad Educativa (Ley orgánica para la mejora de la calidad educativa, 2013), se unen también en esta dirección.

\section{COMPETENCIA MATEMÁTICA}

A efectos de PISA 2015 (OCDE, 2017) la competencia matemática se define como:

La capacidad del individuo para formular, emplear e interpretar las matemáticas en distintos contextos. Incluye razonar matemáticamente y utilizar conceptos, procedimientos, herramientas y hechos matemáticos para describir, explicar y predecir fenómenos. Esto ayuda a las personas a reconocer la presencia de las matemáticas en el mundo y a emitir juicios y decisiones bien fundamentados que necesitan los ciudadanos constructivos, comprometidos y reflexivos (p.64).

En la misma línea, el currículo de la Comunidad Autónoma Vasca Heziberri 2020 define así la competencia matemática: "Aplicar el conocimiento matemático para interpretar, describir, explicar y dar respuestas a problemas relacionados con las necesidades de la vida, utilizando modos de pensamiento, representación y herramientas propias del área" (Dpto. de Educación, Política Lingüística y Cultura, 2016, p. 61). Además, determina los siguientes componentes para la competencia matemática:

1. Identificar y resolver diversas situaciones problemáticas con contenido matemático, aplicando las estrategias pertinentes.

2. Utilizar los distintos conocimientos matemáticos para enfrentarse a situaciones del entorno cotidiano o científico, "modelizando" la situación, formulándola en términos matemáticos, operando con el modelo e interpretando los resultados en el contexto.

3. Interpretar y comunicar informaciones, argumentaciones y resultados.

4. Conocer, relacionar, integrar y valorar los diversos conocimientos matemáticos atendiendo a las características propias de cada situación.

5. Utilizar los distintos modos de razonamiento tanto para justificar las propias conclusiones obtenidas y el proceso seguido como para analizar de forma crítica los resultados presentados por los demás.

6. Seleccionar y utilizar los procedimientos matemáticos adecuados para calcular, representar e interpretar la realidad. 
Para desarrollar la competencia matemática es conveniente que la enseñanza de las matemáticas dote de sentido dentro de un contexto cercano y de interés para el/la estudiante (Gallego et al., 2005; Guirles, 2008; Ojose, 2011). De esta forma, es más sencillo conseguir que el/la aprendiz sea capaz de relacionar las actividades realizadas dentro del aula con situaciones de la vida real, aspecto importante señalado por Boaler (1998) y Alsina (2006) que apunta que de nada sirve acumular conocimientos matemáticos si no se sabe aplicarlos en situaciones reales.

La presentación de una situación-problema abierta, para cuya resolución no haya una estrategia predeterminada, es beneficioso para el/la estudiante. De esta forma, desarrollan la capacidad de afrontar problemas que no tengan una solución única e inminente, que es la realidad a la que se van a tener que enfrentar tanto en la vida cotidiana como en el mundo laboral (Herr, Johnson y Piraro, 2001; Common Core State Standards Initiative, 2010, p. 7). A su vez, el hecho de que haya diversidad en la resolución de los problemas ofrece la oportunidad de que el/la aprendiz comunique y analice los diversos planteamientos y métodos de resolución, dando lugar al lenguaje y razonamiento matemático, actividad fundamental analizado en los artículos (Pugalee, 2001; Jiménez, Suárez y Galindo, 2010; Kostos y Shin, 2010). Godino (2004), por su parte, también remarca que el tipo de comunicación que se da en el aula condiciona el aprendizaje del alumnado y su visión sobre las matemáticas.

\section{APRENDIZAJE BASADO EN PROYECTOS}

A pesar de que haya un consenso sobre la importancia de formar al alumnado en competencias, el nivel de implementación de metodologías adecuadas en las escuelas a menudo es insuficiente y la realidad no refleja los objetivos fijados en los planes de estudio (Bolívar, 2008). Las nuevas metodologías deben cambiar la perspectiva tradicional de transmisión de conocimientos para dotar al alumnado de la capacidad de indagar, investigar y poner en práctica la articulación de los conocimientos adquiridos tal y como se desprende de la práctica competencial (Mateo, 2006). El aprendizaje basado en proyectos o PBL es una de las metodologías que, alejada de exponer a los estudiantes al estrés de los exámenes o de la memorización de tareas, ayuda a generar en el/la estudiante un sentimiento de bienestar y creación de sentido. Según Johari y Bradshaw (2008) el PBL, convierte el aprendizaje del alumnado en un quehacer agradable, esto 
permite construir conocimiento salvaguardando el componente emocional. Además, Mioduser y Betzer (2008) añaden a las cualidades descritas la capacidad que tiene para activar conocimientos previos en el/la aprendiz, para mejorar su razonamiento crítico y la metacognición, así como para desarrollar las destrezas de estudio autónomo y la capacidad de solucionar problemas de manera autónoma. Rodríguez-Sandoval, Vargas-Solano y Luna-Cortés (2010) demostraron, así mismo, que este tipo de metodología consigue mejorar el rendimiento académico entre $30 \%$ y $60 \%$. Un estudio llevado a cabo por Liu, Wang, Tan, Koh y Ee (2009) muestra cómo la percepción sobre el aprendizaje que tienen los estudiantes es más positiva que utilizando otras metodologías, algo que concuerda con el estudio de Willard y Duffrin (2003) quienes descubrieron que este tipo de metodología produce mayores índices de satisfacción entre los y las estudiantes que el sistema tradicional de enseñanza. Larmer, Ross y MergendoIler (2009) también destacan la motivación que manifiesta el alumnado por aprender contenidos o procedimientos que puedan ser aplicados en el proyecto que están llevando a cabo.

Respecto al aprendizaje de las matemáticas las investigaciones indican que mediante el PBL el/la aprendiz interioriza mejor los conceptos matemáticos, retiene de forma prolongada el conocimiento matemático adquirido y logra habilidades para aplicar el conocimiento a situaciones de la vida cotidiana (Gijbels, Dochy, Vanden Bossche y Segers, 2005). Holmes y Hwang (2016) realizaron un estudio con alumnado de secundaria para analizar los efectos del PBL en el aprendizaje de las matemáticas. Destacan que los y las estudiantes que aprenden mediante el PBL presentan mayor motivación y muestra, también, un pensamiento crítico significantemente más alto que los que siguen una metodología de enseñanza tradicional.

\section{OBJETIVO}

Demostrar que mediante el PBL se puede desarrollar adecuadamente la competencia matemática, así como responder a los contenidos curriculares. Con este fin, se presenta un proyecto donde se analiza una tarea rica para la comprensión del concepto de área e introducción del cálculo del área de superficies planas considerándolo útil para la práctica del profesorado de Educación Primaria. 


\section{MÉTODO}

Para esta investigación se ha optado por realizar un estudio de caso desde una perspectiva cualitativa. Este diseño mantiene muchas de las características más representativas del método etnográfico y, si bien, como señalan Lukas y Santiago (2004, p.198), algunos autores: "tratan el estudio de caso como una variante dentro del método etnográfico. Considerándolo como una aplicación del mismo a una unidad de análisis -denominada caso- para conocerla con mayor profundidad". Estos dos autores prefieren diferenciarlo del método etnográfico porque: "aunque en ocasiones pueden coincidir metodológicamente, el objeto de estudio suele ser diferente". En definitiva, el objetivo del estudio de casos, que también es conocido como "estudio del caso único» (Pérez Juste, 1989, p. 479), es lograr un conocimiento profundo de realidades concretas tales como un individuo, una familia, un grupo, una comunidad, una situación o un suceso. Normalmente es una investigación a la que se dedica poco tiempo, pero con una alta intensidad (en general la etnografía suele llevar más tiempo). A estas características, se le suman otras importantes como inductivo (Stake, 1981), holístico y fundamentado en la situación (Guba y Lincoln, 1981; Wilson, 1979); se puede aplicar a situaciones problemáticas y se puede usar para mejorar la práctica (Helmstadter, 1970). Por todo ello, "el estudio de casos es el estudio de la particularidad y de la complejidad de un caso singular, para llegar a comprender su actividad" (Stake, 1998, p. 11), siendo un modelo "valioso para informar de realidades educativas complejas, invisibilidades por la cotidianeidad, para entender procesos internos y descubrir dilemas y contradicciones, ayudando a reflexionar sobre las prácticas educativas" (Álvarez-Álvarez y Fabián Maroto, 2012, p. 5) que brinda la oportunidad de conocer en un corto periodo de tiempo una realidad en profundidad (Karrera, 2008). Es por ello que, para este análisis, en particular, el estudio de caso nos ha permitido indagar en el proceso de enseñanza-aprendizaje basado en la metodología PBL e identificar los principales componentes para desarrollar la competencia matemática dentro de un entorno de educación formal en la etapa de primaria. Con este fin, se han utilizado dos técnicas diferentes de recogida de información: la observación participante y el análisis de las producciones matemáticas del alumnado. La observación participante permite conocer cuál es el problema real que deriva en la necesidad de resolver un problema matemático y cuáles han sido los pasos dados por los y las estudiantes. El registro de la información se realiza mediante las notas de 
campo que se recogen en los diarios de los investigadores y las investigadoras. El registro de las producciones matemáticas se realiza tomando diferentes fotografías de las distintas resoluciones dadas al mismo problema por los diferentes grupos de alumnos y alumnas.

El análisis de contenido permitirá observar cómo se han trabajado los componentes de la competencia matemática y a qué demandas curriculares se ha dado respuesta durante las situaciones-problema que han surgido durante el proyecto. Concretamente, se pone el foco en la situación-problema que requiere el cálculo del área de la manga de un delantal, analizando las diversas estrategias que han empleado los diferentes grupos. Esta información se encuentra dentro de dos de las siete categorías establecidas para el análisis del contenido. La categorización realizada ha sido la siguiente:

- Decisiones iniciales del alumnado

- Instrumentos y medidas necesarias

- Bocetos

- Principales problemas matemáticos

- Propuestas de resolución

- Confección de los delantales

- Presentación

No obstante, para este estudio se ha elegido parte de la información recabada y analizada dentro de las categorías denominadas: "Principales problemas matemáticos" y "Propuestas de resolución" ya que estas dos categorías engloban la mayor parte de la información sobre las estrategias matemáticas empleadas. Todo ello se ha triangulado con los datos recabados durante las sesiones de observación y los registros de las notas de campo y documentos fotográficos. Los resultados de este trabajo se han contrastado con el profesorado del centro educativo y entre los diferentes investigadores que participan en el proyecto de investigación, por lo que los resultados han sido triangulados, dando validez y fiabilidad a la lectura de los resultados tal y como argumenta Arias (2012).

Cabe mencionar que dicha investigación está sujeta al reglamento que especifica el Comité de Ética para investigaciones relacionadas con seres humanos (CEISH) de la UPV/EHU con número de referencia M10/2018/045, de forma que contamos con la aprobación tanto de Antzuola H.E. como de los participantes. 


\section{CONTEXTUALIZACIÓN DE LA INVESTIGACIÓN}

Antzuola H.E. se encuentra en el municipio de Antzuola (País Vasco). La escuela es el único centro escolar de esta localidad guipuzcoana que cuenta con alrededor de 2000 habitantes. Durante el curso escolar cada aula lleva a cabo entre dos o tres proyectos globales con una duración aproximada de 3 o 4 meses. Es el alumnado quien elige el tema del proyecto y realiza una planificación según sus intereses. Partiendo de esta planificación, el profesorado, con la ayuda de una docente del centro que hace las funciones de asesora de los proyectos, diseñan de manera conjunta las tareas que realizan los y las estudiantes. Además, gracias a este trabajo colaborativo entre los profesionales docentes se prevén e implementan las distintas competencias del curriculum, siendo el desarrollo de la competencia matemática un factor importante y de gran interés tomado en cuenta en este diseño pedagógico.

\section{LOS PARTICIPANTES}

En este estudio se observa y analiza las producciones realizadas por las alumnas y los alumnos de sexto de primaria de Antzuola H.E., concretamente, del aula de 6ํA que consta de seis niñas y doce niños de 11-12 años de edad.

\section{EL PROBLEMA}

En este trabajo se describe y analiza una situación-problema que ha tenido lugar en Antzuola H.E. y que ha sido parte del primer proyecto del curso escolar 2017-2018, es decir, se trata de un proyecto cuya elaboración comenzó en septiembre de 2017 y se prolongó hasta enero de 2018. Se ha puesto el foco de interés en una situación-problema que requiere nociones matemáticas para su resolución. Aun así, la situación-problema que se analiza es una actividad englobada en un proyecto interdisciplinar y, por lo tanto, en paralelo a la resolución de este problema se han realizado otras actividades interdisciplinares. 


\section{CONTEXTUALIZACIÓN DE LA SITUACIÓN-PROBLEMA}

El alumnado de 6A de primaria de Antzuola H.E. acordó trabajar el tema de la cocina. Decidieron cocinar algunas recetas y pensaron que sería conveniente crear unos delantales. Cada alumno/a diseñó el boceto de su propio delantal en una hoja DIN A4 detallando las medidas reales que debía tener, ver figura 1.

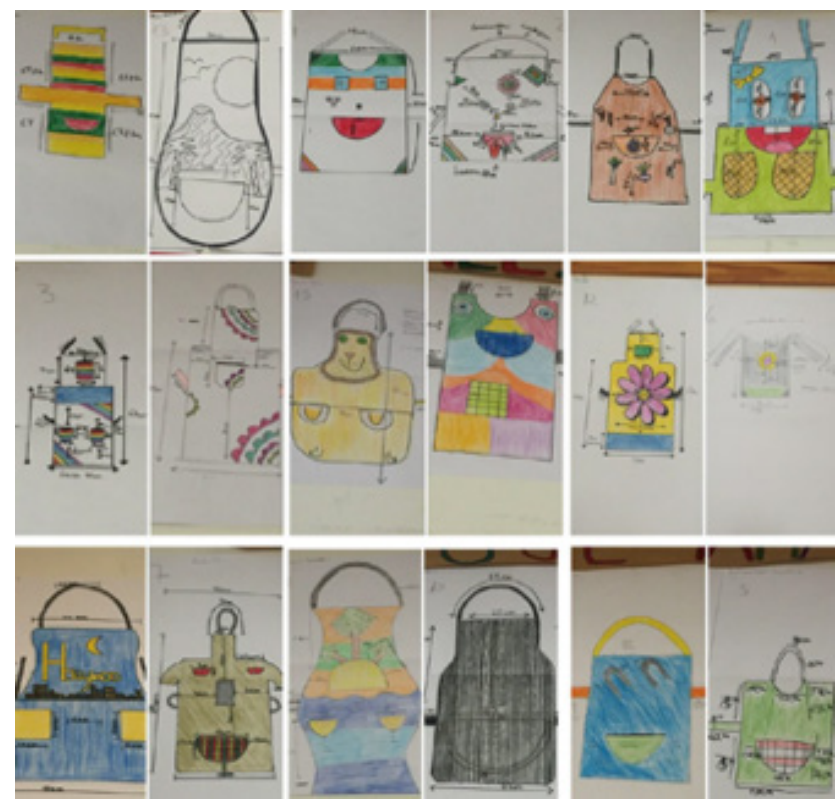

Figura 1. Diseño de los delantales de cada alumno/a con sus correspondientes medidas.

Crear 18 delantales era excesivo y mediante una votación eligieron 3 delantales (el segundo de la primera fila, y el quinto y el sexto de la segunda fila en la figura 1). Los alumnos y alumnas se organizaron en grupos y se les repartió la fotocopia del diseño de un delantal. Cogieron un metro cuadrado de papel y utilizando la regla, el metro y el material que había en el aula de tecnología, dibujaron los patrones en tamaño real. Cortaron y pegaron cada pieza del delantal, dando como resultado los delantales de la figura 2. Aunque la idea era calcular los centímetros cuadrados de cada pieza con el fin de conocer la cantidad de tela que debían comprar para confeccionar los 
delantales, por la complejidad de la actividad y falta de tiempo, ésta se redujo al cálculo del área de la manga de un delantal y fue la profesora quien, finalmente, compró la tela. Una vez comprada la tela pusieron los patrones encima de ésta, los marcaron con una tiza, cortaron los trozos de la tela y cosieron los delantales. Debido a la dificultad que suponía coser las mangas del delantal con forma de camiseta se optó por crear únicamente los otros dos delantales, ver resultado final en la figura 3.
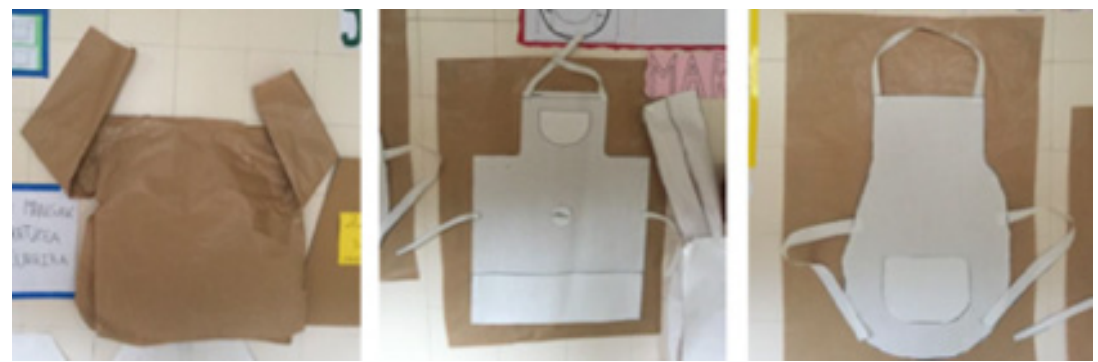

Figura 2. Los delantales seleccionados en papel.
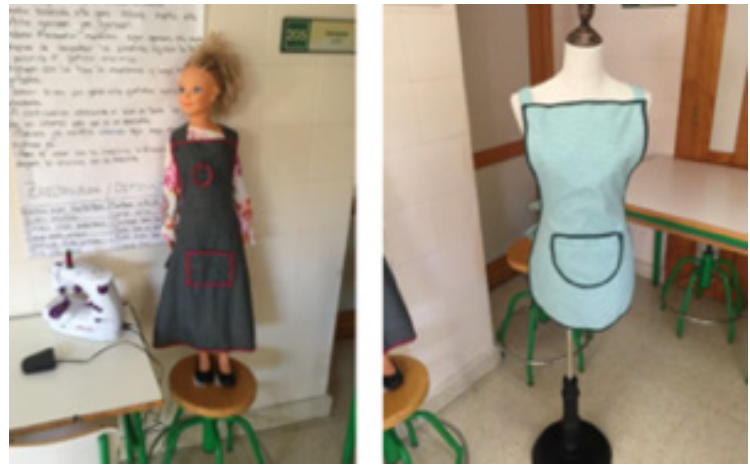

Figura 3. Resultado final de los dos delantales de tela que se cosieron.

\section{DESCRIPCIÓN DE LA SITUACIÓN-PROBLEMA}

Durante la elaboración de los delantales fueron varios los obstáculos que debió superar el alumnado de 6 de primaria. La situación-problema que se describe a continuación, surgió porque necesitaban calcular los metros 
cuadrados de la tela que debían comprar para poder crear los delantales. Por supuesto, teniendo en cuenta que interesaba calcular el área mínima de tela con el objetivo de optimizar el coste y rebajar el presupuesto. Aún así, debido a la complejidad del problema y la falta de tiempo, la profesora decidió simplificar la actividad al cálculo del área de la manga de uno de los delantales.

- Profesora: ¿Cómo podemos saber cuánta tela necesitamos comprar para crear nuestros delantales?

- Mikel: La dependienta ya lo sabrá con ayuda del "equipo" y maquinaria que tiene.

- Profesora: Pero, ¿Si necesitásemos tela para 5000 delantales?

- Sara: iHabrá que calcularlo!

- Profesora: Y ¿Cómo lo vamos a calcular? (silencio)

- Profesora: Os lo pondré más fácil...Si, por ejemplo, queremos calcular el área de esta manga, ¿Cuántos centímetros cuadrados tendrá?

Las alumnas y los alumnos trabajaron por parejas, formando 9 grupos en total, y a cada grupo se le repartió el patrón de la manga en tamaño real, véase la figura 4. A continuación, se describen los métodos de resolución empleados que se han clasificado, finalmente, en 4 categorías por las similitudes que presentan. Estas resoluciones van acompañadas de las elaboraciones originales del alumnado y las aproximaciones en Geogebra creadas por los autores con el fin de facilitar la lectura.

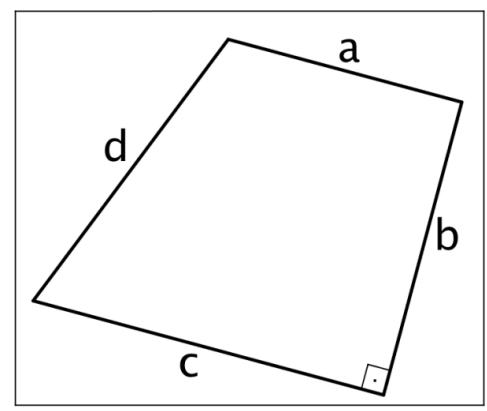

Figura 4. Patrón de la manga que se repartió a cada grupo. 


\section{RESULTADOS: DESCRIPCIÓN Y ANÁLISIS}

\section{DESARROLLO DEL APRENDIZAJE: DESCRIPCIÓN Y ANÁLISIS DE LAS ESTRATEGIAS DE RESOLUCIÓN}

Para calcular el área de la manga los tres grupos incluidos en la Categoría 1 (Grupos 3, 6 y 9) dibujaron centímetros cuadrados en el papel, véase la figura 5.
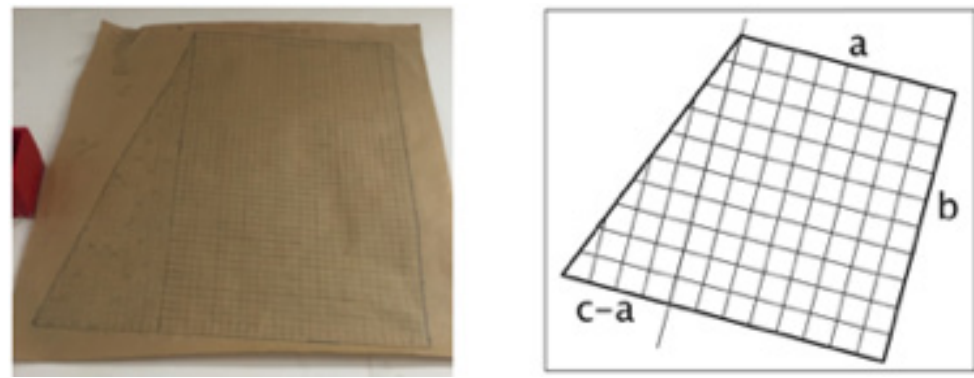

Figura 5. Elaboración original y aproximación con Geogebra (creada por los autores), respectivamente, de un grupo clasificado en la Categoría 1.

Rápidamente se dieron cuenta de que el polígono se podía descomponer en un rectángulo y un triángulo recto. Con la ayuda de los centímetros cuadrados que habían dibujado, dedujeron que podían calcular el área del rectángulo multiplicando la longitud de la base y la altura, $a$ y $b$, respectivamente, en la figura 5, sin necesidad de contar todos los cuadrados. Pero ¿cómo calcular el área del triángulo? Primero, sumaron todos los cuadrados enteros dentro del triángulo. Seguidamente, comenzaron a emparejar los trozos no enteros formando centímetros cuadrados y se percataron de que con dos trozos podían completar un centímetro cuadrado entero. Finalmente, calcularon el área total de la manga mediante la siguiente fórmula:

$$
A_{\text {manga }}=a \cdot b+n^{0} \text { de cuadrados enteros del triángulo }+\frac{n^{0} \text { de cuadrados no enteros del triángulo }}{2} \mathrm{~cm}^{2}
$$

Los dos grupos incluidos en la Categoría 2 (Grupos 1 y 7) descompusieron el polígono de la misma manera que lo hicieron los de la Categoría 1, un rectángulo y un triángulo recto. En cambio, a la hora de calcular el área del rectángulo no trazaron todos los centímetros cuadrados; se limitaron a trazar los 
cuadrados de los bordes para contar cuánto medían los lados a y b, véase la figura 6, y multiplicándolos calcularon el área del rectángulo. En un principio, calcularon el área del triángulo de la misma manera que los de la Categoría 1 (contando los cuadrados enteros y asumiendo que dos trozos no enteros componían un cuadrado). Sin embargo, no quedaron satisfechos con el proceso seguido y le pidieron ayuda a la maestra:

Malen: $\quad$ Creemos que existe otra forma de calcular el área del triángulo.

Profesora: ¿Por qué no trazáis un rectángulo que incluya el triángulo?

Irene: $\quad$ iAh! Convertimos el triángulo en rectángulo, dibujamos los centímetros cuadrados y los dividimos entre dos.

Para calcular el área del rectángulo de base $c-a$ las alumnas necesitaron trazar todos los centímetros cuadrados, véase la figura 6. En esta ocasión, probablemente por la dificultad añadida que conlleva calcular el área del triángulo, no se percataron de que podían calcular el área de ese nuevo rectángulo multiplicando $c-a$ y $b$.
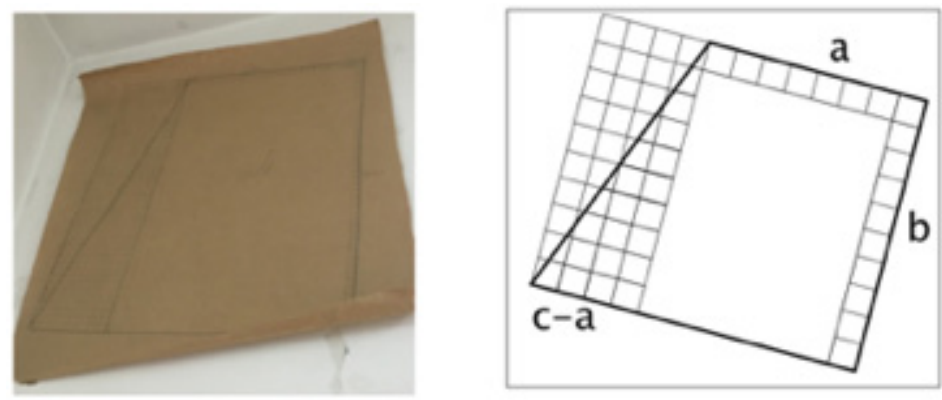

Figura 6. Elaboración original y aproximación con Geogebra (creada por los autores), respectivamente, de un grupo clasificado en la Categoría 2.

Una vez trazados todos los centímetros cuadrados, sumaron todos los cuadrados y los dividieron entre dos obteniendo así el área del triángulo. Finalmente, calculando el área total de la manga mediante la siguiente fórmula:

$$
A_{\text {manga }}=a \cdot b+\frac{n^{\circ} \text { de cuadrados del rectángulo de base } c-a}{2} \mathrm{~cm}^{2}
$$


A tres grupos les ocasionó mucha confusión el hecho de que el lado $b$, véase la figura 4, no fuese paralelo a los lados verticales del papel. Ante esta dificultad, los tres grupos de la Categoría 3 (Grupos 4, 5 y 8) decidieron trazar los centímetros cuadrados tomando como referencia el eje vertical del papel, véase la figura 7.
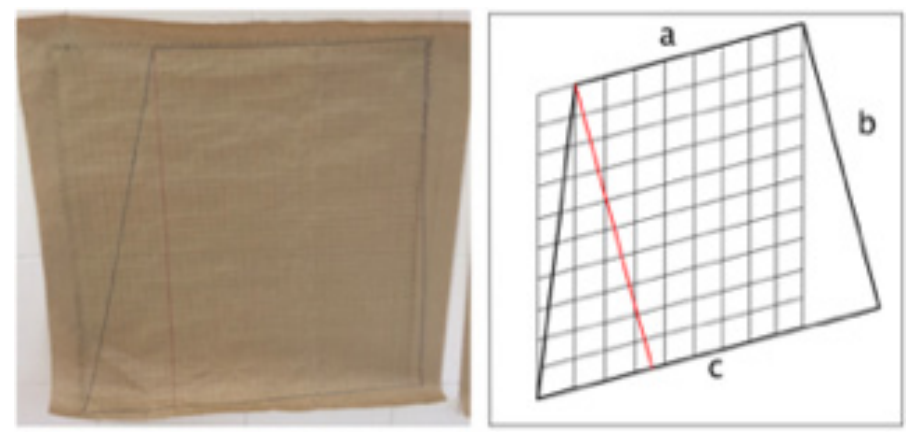

Figura 7. Elaboración original y aproximación con Geogebra (creada por los autores), respectivamente, de un grupo clasificado en la Categoría 3.

Eran conscientes de que dentro del rectángulo que habían trazado quedaba una superficie que no pertenecía a la manga y que debían sustraérselo al área del rectángulo, pero no consiguieron calcular el área de esa superficie extra. Al redactar los pasos que habían seguido escribieron que el área del rectángulo trazado era $1830 \mathrm{~cm}^{2}=18,30 \mathrm{~cm}^{2}$ (dividiéndolo por 100) pero ellos mismos concluyeron que el resultado obtenido era imposible.

El grupo de la Categoría 4 (Grupo 2) comunicó rápidamente que la solución era $172 \mathrm{~cm}^{2}$. Obtuvieron la solución sumando la longitud de los lados del polígono $a, b, c y d$ sin percatarse de que lo que habían calculado era el perímetro del trapecio. Este error, la confusión entre los conceptos de área y perímetro, es un error muy común entre los y las estudiantes de hasta 13-14 años, así lo demuestran las investigaciones (Mántica, del Maso, Götte y Marzioni, 2002). La maestra les propuso que confirmaran el resultado y comenzaron a trazar los centímetros cuadrados comprobando que había más de $172 \mathrm{~cm}^{2}$. De esta manera, las alumnas y los alumnos, enfrentados a un problema contextualizado, pudieron comprobar el error cometido.

Este grupo fue el único que comenzó a trazar los centímetros cuadrados desde el lado del trapecio que no formaba $90^{\circ}$ con ninguno de los dos lados contiguos 
obteniendo así paralelogramos de un centímetro cuadrado de área, véase la figura 8.

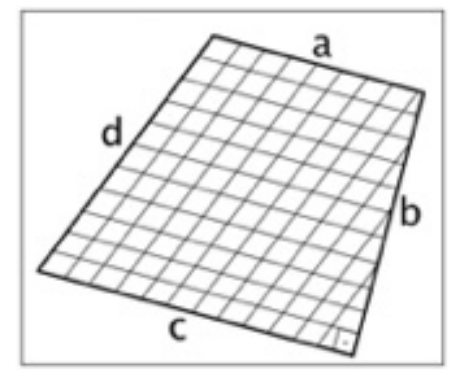

Figura 8. Primera elaboración de la Categoría 4 con Geogebra (creada por los autores).

Una vez llegados a este punto se bloquearon, observaron los trabajos del resto de grupos y decidieron comenzar a trazar los cuadrados tomando como referencia el lado vertical del papel. La propuesta de uno de los participantes:

losu: $\quad$ Si le damos forma de cuadrado a la figura lograremos la respuesta.

Profesora: De acuerdo, pero tened en cuenta que nosotros solamente buscamos el área de la figura y, por lo tanto, habrá que restar lo de fuera. Tendréis que pensar cómo.

Se encontraron con las mismas dificultades que los de la Categoría 3 y siguieron la misma estrategia de resolución, véase la figura 9.

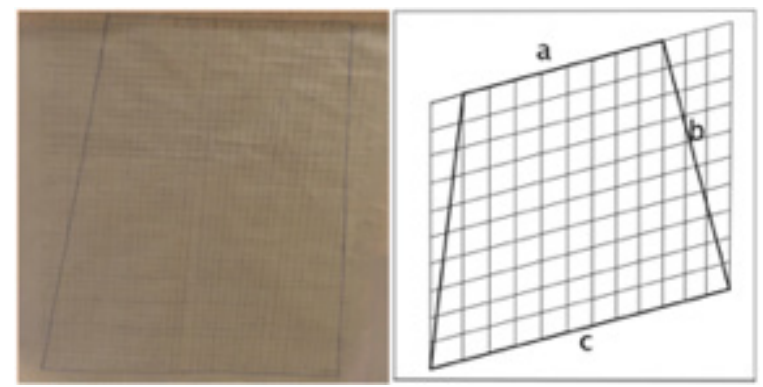

Figura 9. Elaboración original y aproximación con Geogebra (creada por los autores), respectivamente, de un grupo clasificado en la Categoría 4. 
A modo de conclusión del análisis de las estrategias de resolución, podemos resaltar que todos los grupos han iniciado el proceso del cálculo del área cubriendo el trapecio con una unidad de superficie. Kordaki y Potari (2002) creen imprescindible esta representación visual del área para una buena comprensión del concepto. Los grupos incluidos en la Categoría 1 y 2 han descompuesto el trapecio en dos figuras planas: un triángulo recto y un rectángulo. Según Chamorro (1997) esta estrategia es importante y debería considerarse en el proceso de aprendizaje del cálculo de superficies. Además, la representación de las unidades de superficie ha ayudado al alumnado a deducir las fórmulas del rectángulo y triángulo. Del Olmo, Moreno y Gil (1989) defienden que ese debe ser el procedimiento "la fórmula como un último paso, como un camino más corto para lograr el resultado que se ha obtenido antes por medios más espontáneos y laboriosos" (p. 46). En definitiva, los y las estudiantes han tratado el tema del área desde un punto de vista cualitativo y, como estiman conveniente las investigaciones (Mántica, del Maso, Götte y Marzioni, 2002; Marmolejo y González, 2015), dejando al margen la introducción precoz de las fórmulas y el simple tratamiento cuantitativo.

\section{DISCUSIÓN DE LOS RESULTADOS Y CONCLUSIONES}

Si examinamos el currículo Heziberri 2020 de la Comunidad Autónoma Vasca (Dpto. de Educación, Política Lingüística y Cultura, 2016) observaremos que mediante el proyecto de la Cocina se han abarcado varios de los contenidos del Bloque 3 de La Medida y el Bloque 4 de la Geometría. Al trazar los bocetos de los delantales cada alumno/a tuvo que estimar la medida y señalar la unidad más adecuada. Al crear los patrones del delantal tuvieron que interpretar el texto numérico del boceto creado por alguno/a de sus compañeros/as y, basándose en esas medidas, dibujar su propio patrón en tamaño real. El cálculo del área de la manga dio pie, por un lado, a recordar el concepto del perímetro, interiorizar el concepto de área, y restar y sumar superficies en situaciones reales y, por otro lado, a la introducción de figuras planas, a la clasificación de triángulos y cuadriláteros según sus lados y a la descomposición de una figura plana en otras dos. Todo ello trabajando en grupo, con el aliciente de tener que transmitir correctamente las ideas utilizando un vocabulario apropiado, para que hubiera una comunicación adecuada entre todos los participantes. 
En cuanto a la competencia matemática, observamos que se han abordado los componentes determinados en el apartado del Marco Teórico. 1) Cada grupo ha ideado su propia estrategia para el cálculo del área de la manga con forma de trapecio como se ha mostrado en el apartado anterior; 2) Todos los grupos han formulado el problema en términos geométricos haciendo uso de los centímetros cuadrados que han dibujado, han calculado el área del trapecio (algunos por conteo, otros concluyendo la fórmula de área) e interpretado los resultados que informan acerca de la cantidad de tela que se necesita comprar para una manga. Ejemplos claros de esto son las conclusiones de los grupos clasificados en las Categorías 3 y 4 que al obtener que el área del rectángulo era de 18,30 y 172 respectivamente, dedujeron que habían cometido algún error. 3) Al trazar los bocetos de los delantales han comunicado las medidas y al crear los patrones han tenido que interpretar esas medidas. Además, como han trabajado de forma grupal en el cálculo del área, se han visto obligados a comunicar con precisión sus argumentos y a interpretar los de los demás compañeros/ as; 4) En la introducción de la situación-problema, la profesora guía al alumnado con el fin de que relacionen la cantidad de tela, superficie, que necesitan comprar con el concepto de área. Partiendo de esta situación, ellos/as mismos/ as descomponen el patrón de la manga que se les facilita en unidades de medida con el fin de calcular el área. 5) Durante el proceso de resolución, trabajando en grupo, ha sido imprescindible para los y las estudiantes razonar y justificar constantemente las ideas de cada uno/a y analizar de forma crítica las propuestas de los/as compañeros/as. Además, en Antzuola H.E. es parte de la filosofía del centro que tras cada actividad las alumnas y los alumnos, individualmente o en grupo, resuman en una hoja el proceso seguido y lo compartan con el resto de la clase, lo que implica tener que comunicar las propias ideas de la mejor forma posible y escuchar las ideas de los demás de forma crítica. A su vez, de este modo, se hace una puesta en común de los distintos modos de razonamiento que se han seguido; 6) Teniendo en cuenta que es un problema planteado en $6^{\circ}$ de primaria, el hecho de descomponer el trapecio en un triángulo recto y un rectángulo ha sido un procedimiento matemático adecuado para representar el problema y resolverlo.

De esta forma, se demuestra que mediante el aprendizaje basado en proyectos se están formando ciudadanos matemáticamente competentes. El alumnado, para dar solución a los problemas matemáticos que le surgen, hace uso de la información que conoce y se basa en las nociones matemáticas que domina aplicando el razonamiento lógico-matemático. Como ya han señalado otros 
autores (Vaydia, 2015; Marmolejo y González, 2015) esta forma de trabajar fomenta la creatividad; el alumnado es libre de elegir la estrategia de resolución que quiere seguir, sin la imposición de una instrucción. El mercado laboral cada vez demanda trabajadores más autónomos capaces de dar respuesta a los problemas que se generan en el día a día (Hidalgo y León, 2006). La capacidad de generar conocimiento se ha convertido indispensable en la nueva sociedad del conocimiento, convirtiendo el capital intelectual de la sociedad en un activo estratégico de las empresas. Los estamentos sociales cada vez se basan más en el conocimiento y menos en la imposición y, por consiguiente, las empresas que basan sus relaciones en el diálogo y en la cooperación están obteniendo mejores resultados (Gómez, Latorre, Sánchez y Flecha, 2006, p.39).

\section{REFERENCIAS}

Alsina, Á. (2006). Desarrollo de competencias matemáticas con recursos lúdico-manipulativos. Narcea.

Álvarez-Álvarez, C. y Fabián-Maroto, J. L. (2012). La elección del estudio de caso en investigación educativa. Gazeta de Antropología, 28(1), 1-13.

Antzuola Herri Eskola. (2006). El viaje a Marte. Cuadernos de Pedagogía, 355, 55-58.

Arias, F. (2012). El proyecto de investigación. 6ํe edición. Ca. Editorial Episteme Venezuela. Boaler, J. (1998). Open and closed mathematics: Student experiences and understandings. Journal for Research in Mathematics Education, 29, 41-62.

Bolívar, A. (2008). El discurso de las competencias en España: educación básica y educación superior. Revista de Docencia Universitaria, 6(2), 1-23.

Chamorro, M. C. (1997). Estudio de las situaciones de enseñanza de la medida en la escuela elemental (Tesis de doctorado no publicada). UNED.

Common Core State Standards Initiative (CCSSI). (2010). Common Core State Standards for Mathematics. National Governors Association Center for Best Practices and the Council of Chief State School Officers.

Del Olmo, M. A. Moreno, M. F. y Gil, F. (1989). Superficies y volumen. ¿̇Algo más que el trabajo con fórmulas? Síntesis.

Departamento de Educación, Política Lingüística y Cultura. (2016). Currículo de la Educación Básica.

Departamento de Educación, Política Lingüística y Cultura. (s.f.-a). Caracterización de la situación problema. 
Departamento de Educación, Política Lingüística y Cultura. (s.f.-b). Heziberri 2020, Marco del Modelo Educativo Pedagógico.

Gallego, C., Pons, M., Alemany, M., Barceló, M., Guerra, M., Orfila, M., Pons, C., Pons, F., Pons, F. C., Pons, T., y Triay, N. (2005). Repensar el aprendizaje de las matemáticas. Matemáticas para convivir comprendiendo el mundo. Graó.

Gijbels, D., Dochy, F., Vanden Bossche, P. y Segers, N. (2005). Effect of problem based learning: A meta-analysis from the angle of assessment. Review of Educational Research, 75, 27-61.

Godino, J. D. (2004). Hacia una teoría de la instrucción matemática significativa. Documento de trabajo del curso de doctorado "Teoría de la educación Matemática". Departamento de Didáctica de la Matemática. Universidad de Granada.

Gómez, J., Latorre, A., Sánchez, M., y Flecha, R. (2006). Metodología comunicativa crítica. El Roure.

Guba y Lincoln, 1981. Effective evaluation: improving the usefulness of evaluation results through responsive and naturalistic approaches. Jossey-Bass.

Guirles, J. R. G. (2008). Competencia Matemática en Primaria. Sigma, 32, 31-49.

Helmstadter, G. C. (1970). Research concepts in human behavior: Education, psychology, sociology. Appleton-Century-Crofts.

Herr, T., Johnson, K., y Piraro, D. (2001). Problem solving strategies: Crossing the river with dogs and other mathematical adventures. Key Curriculum Press.

Hidalgo, A., y León, G. (2006). La importancia del conocimiento científico y tecnológico en el proceso innovador. Revista madri+d, 39(1).

Holmes, V. L. y Hwang, Y. (2016). Exploring the effects of project-based learning in secondary mathematics education. The Journal of Educational Research, 109(5), 449-463.

Jiménez, A., Suárez, N. Y. y Galindo, S. M. (2010). La comunicación eje en la clase de matemáticas. Praxis y Saber, 1(2), 173-202.

Johari, A. y Bradshaw, A. C. (2008), Project-based learning in an internship program: A qualitative study of related roles and their motivational attributes. Educational Technology Research and Development, 56(3), 329-359.

Karrera, I. (2008). El método cualitativo como herramienta válida en la resolución de conflictos escolares (Tesis doctoral no publicada) UPV/EHU.

Kostos, K. y Shin, E. (2010). Using math journals to enhance second graders' communication of mathematical thinking. Early Chilhood Education Journal, 38, 223-231.

Kordaki, M. y Potari, D. (2002). The effect of area measurement tools on student strategies: the role of a computer microworld, International Journal of Computers for Mathematical Learning, 7(1), 1-36. 
Larmer, J., Ross, D. y Mergendoller, J. (2009). PBL starter kit: To-the-point advice, tools and tips for your first project in middle or high school. Unicorn printing Specialists.

Ley orgánica para la mejora de la calidad educativa (LOMCE) (Ley Orgánica 8/2013, 9 de diciembre). Boletín Oficial del Estado, núm. 295, 2013, 10 diciembre.

Liu, W. C., Wang, C. K. J., Tan, O. S., Koh, C. y Ee, J. (2009). A self-determination approach to understanding students' motivation in project work. Learning and Individual Differences, 19(1), 139-145.

Lukas, J. F. y Santiago, K. (2004). Evaluación Educativa. Alianza.

Mántica, A. M, del Maso, M. S., Götte, M. y Marzioni, A. (2002). La confusión entre área y perímetro. Análisis de una propuesta áulica. Educación Matemática, 14(1), 111-119. http:// www.revista-educacion-matematica.org.mx/descargas/Vol14-1.pdf

Mateo, J. L. (2006). Sociedad del conocimiento. Arbor, 182(718), 145-151.

Marmolejo, G. A. y González, M. T. (2015). El área de superficies planas en el campo de la educación matemática. Estado de la cuestión. Revista Electrónica de Investigación en Educación en Ciencias, 10(1), 45-57.

Mioduser, D. y Betzer, N. (2008). The contribution of project-based-learning to high-achievers' acquisition of technological knowledge and skills. International Journal of Technology and Design Education, 18(1), 59-77.

OCDE (2017), Marco de Evaluación y de Análisis de PISA para el Desarrollo: Lectura, matemáticas y ciencias, Versión preliminar, OECD Publishing. https://www.oecd.org/ pisa/aboutpisa/ebook\%20-\%20PISA-D\%20Framework_PRELIMINARY\%20version_ SPANISH.pdf

Ojose, B. (2011). Mathematics Literacy: are we able to put the mathematics we learn into everyday use? Journal of Mathematics Education, 4(1), 89-100.

Pérez Juste, R. (1989). Los datos para la evaluación. En R. Pérez Juste y J.M. García Ramos, Diagnóstico, evaluación y toma de decisiones. Rialp.

Pugalee, D. K. (2001). Using communication to develop students' mathematical literacy. Mathematics teaching in middle school, 6(5), 296-299.

Recomendación del Parlamento Europeo y del Consejo, de 18 de diciembre de 2006, sobre las competencias clave para el aprendizaje permanente (2006/962/CE), 18 de diciembre de 2006. https://eur-lex.europa.eu/legal-content/ES/TXT/PDF/?uri=CELEX:32006H0962\&from=ES

Rodríguez-Sandoval, E., Vargas-Solano É. M. y Luna-Cortés, J. (2010). Evaluación de la estrategia, "aprendizaje basado en proyectos". Educación y educadores, 13(1), 13-25. Sasieta, E., y Etxaniz, P. (2015). He estado pensando y mi cabeza me dice que... Revista Aula de Innovación Educativa, 238, 24-28. 
Stake, R. E. (1981). Setting standards for educational evaluators. Evaluation News, 2(2), 148-152.

Vaydia, A. (2015). Using Project- and Theme-Based Learning to Encourage Creativity in Science. Journal of College Science Teaching, 45(2).

Walker, A. (2015). Essential readings in problem-based learning: Exploring and extending the legacy of Howard S. Barrows. Purdue University Press.

Willard, A. K. y Duffrin, M. W. (2003). Utilizing project-based learning and competition to develop student skills and interest in producing quality food items. Journal of Food Science Education, 2(4), 69-73.

Wilson, S. (1979). Explorations of the usefulness of case study evaluations. Evaluation Quarterly, 3(3), 446-459.

ANE IZAGIRRE

Dirección: Plaza Oñati, 3. 20018. Donostia

Teléfono: $\quad 943017050$ 